Article

\title{
Application of Direct Numerical Simulation to Determine the Correlation Describing Friction Losses during the Transverse Flow of Fluid in Hexagonal Array Pin Bundles
}

\author{
Yury E. Shvetsov ${ }^{1, *}$, Yury S. Khomyakov ${ }^{1}$, Mikhail V. Bayaskhalanov ${ }^{2, *}$ and Regina P. Dichina ${ }^{2}$ \\ 1 Department of Science, Joint Stock Company "PRORYV”, 107140 Moscow, Russia; hus@proryv2020.ru \\ 2 Department of Thermal Physics, Institute of Nuclear Physics and Engineering, National Research Nuclear \\ University MEPhI (Moscow Engineering Physics Institute), 115409 Moscow, Russia; \\ regina.dichina@yandex.ru \\ * Correspondence: shuye@proryv2020.ru (Y.E.S.); mvbayaskhalanov@mephi.ru (M.V.B.)
}

check for updates

Citation: Shvetsov, Y.E.; Khomyakov, Y.S.; Bayaskhalanov, M.V.; Dichina, R.P. Application of Direct Numerical Simulation to Determine the Correlation Describing Friction Losses during the Transverse Flow of Fluid in Hexagonal Array Pin Bundles. Fluids 2022, 7, 22. https:// doi.org/10.3390/fluids7010022

Academic Editor: D. Andrew S. Rees

Received: 5 December 2021

Accepted: 27 December 2021

Published: 3 January 2022

Publisher's Note: MDPI stays neutral with regard to jurisdictional claims in published maps and institutional affiliations.

Copyright: (C) 2022 by the authors. Licensee MDPI, Basel, Switzerland. This article is an open access article distributed under the terms and conditions of the Creative Commons Attribution (CC BY) license (https:// creativecommons.org/licenses/by/ $4.0 /)$.

\begin{abstract}
This paper presents the results of a numerical simulation to determine the hydraulic resistance for a transverse flow through the bundle of hexagonal rods. The calculations were carried out using the precision CFD code CONV-3D, intended for direct numerical simulation of the flow of an incompressible fluid (DNS-approximation) in the parts of fast reactors cooled by liquid metal. The obtained dependencies of the pressure drop and the coefficient of anisotropy of friction on the Reynolds number can be used in the thermal-hydraulic codes that require modeling of the flow in similar structures and, in particular, in the inter-wrapper space of the reactor core.
\end{abstract}

Keywords: direct numerical simulation; fast breeder reactor; liquid metal; hydrodynamic

\section{Introduction}

Currently under development are various liquid metal cooled nuclear reactor projects related to Generation IV reactors. Improving the calculation accuracy when justifying the thermohydraulic characteristics of a fast reactor is an important applied problem, since this can affect its reliability and operational safety. On the other hand, the refinement of the calculation makes it possible to reduce the degree of conservatism in the design. One of the design features of a typical fast reactor with fuel assemblies of cassette type in the core is the presence of an inter-wrapper space (IWS) in the core. This actually means the presence of, simultaneously, two coolant flows in the reactor core: one of them is the main one, passing inside the fuel assembly and responsible for heat removal from the fuel rods, and the other, passing through the IWS and usually making up a few percent of the total flow through the reactor. Both streams, in the course of their movement, exchange heat through the fuel assembly. The degree of influence of the processes in the IWS on the parameters of the reactor and the core as a whole depends on the design features of the reactor and the investigated mode of its operation. This influence was considered in [1].

The fraction of heat removed from the core through the IWS in the rated power mode of the reactor is usually small, but even in this case, the spatial non-uniformity of the velocity field in the IWS can noticeably affect the temperatures in some fuel sub-assembles of the core. The influence of processes in the inter-wrapper space on heat transfer in the core increases with primary flow rate decrease in the reactor. The need to take into account the influence of IWS arises when studying transient modes and some beyond design basis accidents. For example, the key point in the study of a beyond design basis accident caused by the loss of flow in the primary and secondary circuits of the reactor with a simultaneous failure of the emergency protection system is the possibility of more accurate modeling of the effects of reactivity, and, in particular, the effect associated with the shape change of the core during heating. In this case, the picture of the bends of the fuel assemblies 
largely depends on the azimuthal temperature gradients over the cross section of the fuel assembly wrapper, which, in turn, depend on variation of the spatial temperature distribution of sodium that washes the outer surface of the wrapper. Adequate modeling of the thermo-hydrodynamic processes in the IWS is also important when investigating the ULOF accident. The sodium boiling in the IWS can make a significant contribution to the reactivity, and seriously affect the course of the accident and its consequences.

The influence of processes in the inter-wrapper space on heat transfer in the core is most clearly manifested in the emergency heat removal mode, when there is no forced circulation along the circuits, and a significant fraction of the heat of the decay heat released in the fuel elements is removed due to the circulation of sodium in the IWS. This effect becomes especially significant in the design version of a fast reactor equipped with decay heat exchangers immersed in the upper reactor plenum. In this case, the "cold" coolant from the outlet windows of the decay heat exchanger is discharged first into the upper plenum of the reactor, and then into the peripheral part of the inter-wrapper space, where it first moves in a radial direction to the center of the core, where it then heats up and forms an ascending flow. As a result, the efficiency of heat removal from the core and the prevention of emergency overheating of fuel elements is largely determined by the resistance to the flow during its horizontal movement towards the center in the IWS.

Justification of nuclear reactors is currently carried out mainly using computational codes of varying degrees of detail. Full CFD modeling of a nuclear reactor, taking into account all the features of its flow path, requires unrealistically large expenditures of computer resources, therefore, in practice, the porous medium approximation is often used to describe heat transfer in separate parts of the reactor. At the same time, detailed modeling of the flow and heat exchange in such small structures as the inter-pin or interwrapper space is abandoned, and the interaction with the "framework" of the porous medium is described integrally by introducing mass friction forces into the momentum equations. These friction forces in pin-bundle structures depend on the flow direction; therefore, the corresponding friction coefficients have anisotropy. Additionally, if the empirical correlations for determining the friction coefficients for rod bundles, both in the longitudinal and transverse directions are available, for example [2], then there are no such correlations for calculating of friction coefficients for the transverse flow of liquid in rod bundles formed by hexagonal cylinders.

Currently, the idea of replacing full-scale experiments by calculations using precision codes is actively developing, The use of such codes makes it possible to check the possible flow modes in various equipment of the reactor plant in a wide range of parameters, without resorting to the need to construct experimental facility. The obtained results can be used to develop correlations describing various processes of heat and mass transfer in the core and circuit elements. The correlations can later be used in engineer codes that use the subchannel technique or the porous medium approximation.

It is proposed to implement this approach and to use the precision scalable eddyresolving CFD code CONV-3D [3,4]. The code is developed at the Nuclear Safety Institute of the Russian Academy of Sciences (IBRAE) and based on the DNS approximation. CONV-3D code was validated against the wide set of thermal-hydraulic experiments [5,6]. The code is intended for performing precision thermo-hydrodynamic calculations in fuel assemblies, core, and other sections of fast reactor circuits. The computational core of the program is based on the finite volume method (FVM) and is used to solve the Navier-Stokes equation with both constant and variable coefficients. The program allows us to carry out calculations on Cartesian and IBM grids.

Thermo-hydrodynamic processes in the CONV-3D are modeled using nonstationary Navier-Stokes equations in natural variables together with the energy equation [3-5]:

$$
\frac{d \rho u}{d t}=-\frac{\partial p}{\partial x}+\frac{\partial}{\partial x} \sigma_{x x}+\frac{\partial}{\partial y} \sigma_{x y}+\frac{\partial}{\partial z} \sigma_{x z}+\rho g_{x}
$$




$$
\begin{gathered}
\frac{d \rho v}{d t}=-\frac{\partial p}{\partial y}+\frac{\partial}{\partial x} \sigma_{y x}+\frac{\partial}{\partial y} \sigma_{y y}+\frac{\partial}{\partial z} \sigma_{y z}+\rho g_{y} \\
\frac{d \rho w}{d t}=-\frac{\partial p}{\partial z}+\frac{\partial}{\partial x} \sigma_{z x}+\frac{\partial}{\partial y} \sigma_{z y}+\frac{\partial}{\partial z} \sigma_{z z}+\rho g_{z} \\
\operatorname{div} \mathbf{v}=0 \\
\frac{d \rho H}{d t}+\operatorname{div}(\rho \mathbf{v} H)=\operatorname{div}(\operatorname{kgrad} T)
\end{gathered}
$$

where $\rho$ is the density; $u, v$ and $w$ are the components of the velocity vector; $p$ is the pressure; $\sigma_{i j}$ are the symmetric components of the symmetric viscous-stress tensor; $g_{i}$ are the components of the gravitational force vector; $t$ is the time; $\mathbf{v}=(u, v, w)$ is the velocity vector; $H=\int_{T_{0}}^{T} \mathrm{c}(\xi) d \xi$ is the enthalpy; $\kappa$ is the thermal conductivity; $T$ is the temperature; $T_{0}$ is the initial temperature; $c$ is the specific heat;

The numerical implementation of splitting schemes for the Navier-Stokes equations in the CONV-3D is performed in the operator-difference formulation as a two-step procedure with pressure adjustment. To obtain a time-integrated scheme for the energy equation, the operators of the equation are split into two parts associated with the enthalpy and temperature, which gives a two-step procedure. The operators in the equation of motion are also split into two parts: the transfer of velocity by convection/diffusion, a pressure gradient. More details about the numerical algorithm implemented in the CONV-3D are outlined in [4].

\section{Problem Statement}

The design area was a rectangular domain of the reactor core assembled from fuel assemblies and cooled by liquid sodium. Taking into account the high requirements of the CONV-3D code for computing power and assuming the absence of flow in the vertical direction, the computational domain was constructed as a prism of small thickness (Figure 1). The design area consisted of 11 complete and 12 incomplete hexagons.

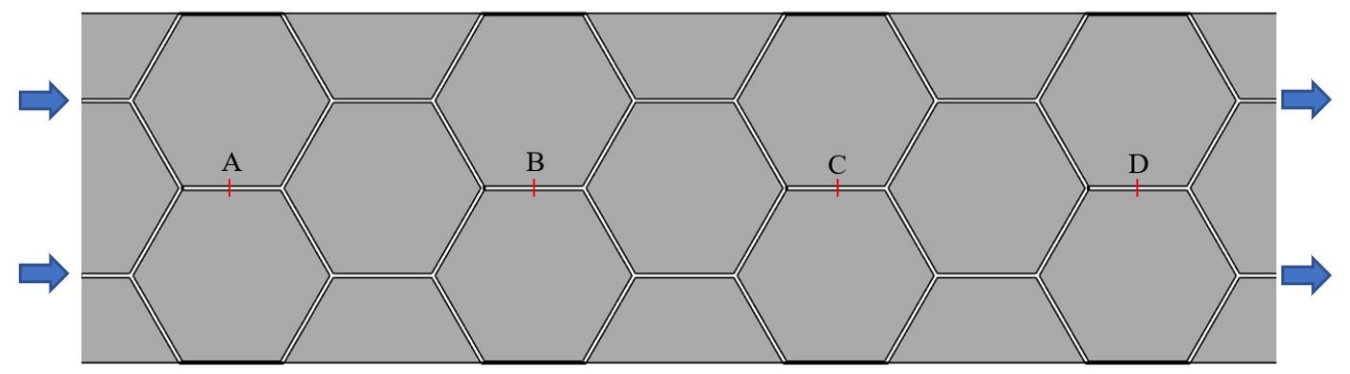

Figure 1. Calculation area and cross-sections for measuring pressure drop.

The fuel assembly width across flats was equal to $181 \mathrm{~mm}$, and the size of the intercassette space was up to $5 \mathrm{~mm}$. The sodium coolant temperature was maintained equal to $723 \mathrm{~K}$. The parametric calculations were performed, where the sodium flow rate at the domain inlet was varied in the range of $0.25-2 \mathrm{~m} / \mathrm{s}$.

\section{Computational Mesh and Boundary Conditions}

The geometry of the computational domain (Figure 2) was constructed using the Geometry Editor embedded in the code. As boundary conditions, slip conditions were set on the lateral, lower and upper surfaces of a rectangular prism, and "pumps" with positive and negative flow rates were set at the inlet and outlet of the design area, which provided a constant flow simulation. The meshing of the computational domain consisted of 4099 cells in the longitudinal direction, 1025 cells in the transverse direction and 9 cells in the vertical direction. All calculations were carried out on a SENPAI computing cluster, (4 nodes, 160 cores, 1.5 TB RAM, 8 TB HDD, 10 TFLOPS, Intel ${ }^{\circledR}$ Xeon ${ }^{\circledR}$ Gold 6230 CPU 
2.1 GHz) with parallelization into 32 physical cores. The time spent on one iteration was approximately $10 \mathrm{~s}$.

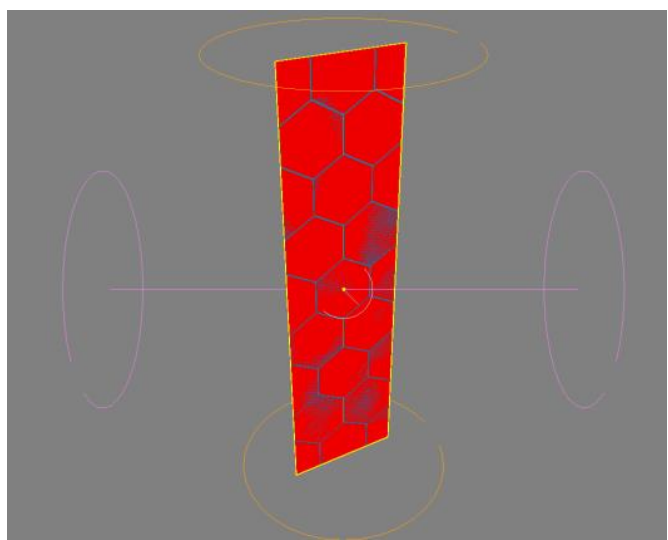

Figure 2. General view of the geometry of the computational domain.

The calculation began with an initial approach and continued until the stationary regime was reached. The solution was considered as steady after calculation, during a time interval that was equal to double transport time of passing the domain by coolant. Then, one more transport interval was calculated for the control of stability and calculations of time-averaged parameters of the flow.

\section{Calculation Results}

Eight calculations were performed with different values of the coolant velocity at the entrance to the computational domain. For each series of calculations, the pressure drop was determined between sections A and B, B and C, C and D, as shown on Figure 1 .

The calculated pressure drops for various values of the flow rate at the entrance are presented in the Table 1.

Table 1. Pressure drops between sections.

\begin{tabular}{ccccc}
\hline $\boldsymbol{v}, \mathbf{m} / \mathbf{s}$ & $\mathbf{R e}$ & $\Delta p_{\text {A-B }}, \mathbf{P a}$ & $\Delta p_{\text {B-C }}, \mathbf{P a}$ & $\Delta p_{\mathrm{C}-\mathrm{D}}, \mathbf{P a}$ \\
\hline 0.264 & 8550 & 383 & 368 & 380 \\
\hline 0.529 & 17,133 & 2194 & 2117 & 2186 \\
\hline 0.795 & 25,748 & 5493 & 5293 & 5470 \\
\hline 1.06 & 34,330 & 10,248 & 9870 & 10,213 \\
\hline 1.326 & 42,945 & 16,821 & 16,282 & 16,747 \\
\hline 1.592 & 51,560 & 24,671 & 23,864 & 24,544 \\
\hline 1.854 & 60,046 & 34,182 & 33,047 & 34,127 \\
\hline 2.118 & 68,596 & 45,014 & 43,728 & 44,902 \\
\hline
\end{tabular}

The influence of the boundary conditions can be seen from the results, since the pressure drop in sections $\mathrm{A}-\mathrm{B}$ and $\mathrm{C}-\mathrm{D}$ differs from the difference between sections $\mathrm{B}$ and C. However, the deviation in the entire investigated range of Reynolds numbers is less than $3 \%$.

The coefficient of anisotropy of friction in the bundles is usually defined as the ratio of pressure drops:

$$
K_{r z}=\Delta p_{r} / \Delta p_{z}
$$

where $\Delta p_{r}$ and $\Delta p_{z}$ indicate the pressure drops per unit length in the transverse and longitudinal directions, respectively. 
Calculated with CONV-3D, the pressure drops $\Delta \mathrm{p}_{\mathrm{r}}$ were compared with the pressure losses $\Delta p_{z}$, calculated using the known empirical relationship [2] to determine the hydraulic resistance of a flat channel:

$$
\Delta p_{z}=1.1 \cdot \xi \cdot \frac{l}{d_{\mathrm{H}}} \cdot \frac{\rho \cdot u^{2}}{2}
$$

where $\xi=0.3164 / R e^{0.25}$ and hydraulic diameter $d_{H}$ is equal to double width of inter cassette gap.

The results of calculating the coefficient of friction anisotropy from the Reynolds number are presented in Table 2.

Table 2. Dependence of the coefficient of friction anisotropy on the Reynolds number.

\begin{tabular}{ccccc}
\hline $\mathbf{R e}$ & $\boldsymbol{\Delta} \boldsymbol{p}_{\boldsymbol{z}}, \mathbf{P a}$ & $\boldsymbol{K}_{\boldsymbol{r} z \mathbf{A}-\mathbf{B}}$ & $\boldsymbol{K}_{r z \mathbf{B}-\mathrm{C}}$ & $\mathbf{K}_{\mathbf{r z C}-\mathbf{D}}$ \\
\hline 8550 & 34 & 11.22 & 10.77 & 11.12 \\
\hline 17,133 & 115 & 19.03 & 18.36 & 18.96 \\
\hline 25,748 & 235 & 23.36 & 22.51 & 23.26 \\
\hline 34,330 & 389 & 26.34 & 25.37 & 26.25 \\
\hline 42,945 & 576 & 29.22 & 28.29 & 29.09 \\
\hline 51,560 & 793 & 31.12 & 30.11 & 30.96 \\
\hline 60,046 & 1035 & 33.03 & 31.93 & 32.98 \\
\hline 68,596 & 1306 & 34.46 & 33.47 & 34.37 \\
\hline
\end{tabular}

The results of calculating of the friction anisotropy coefficient versus Re number are approximated by a polynomial of the 2nd degree:

$$
K_{r z}(\operatorname{Re})=4.532+8.518 \cdot 10^{-4} \cdot \operatorname{Re}-6.314 \cdot 10^{-9} \cdot \operatorname{Re}^{2}
$$

and are shown on Figure 3. The maximum deviation of the approximating curve from the calculated values is less than $6 \%$.

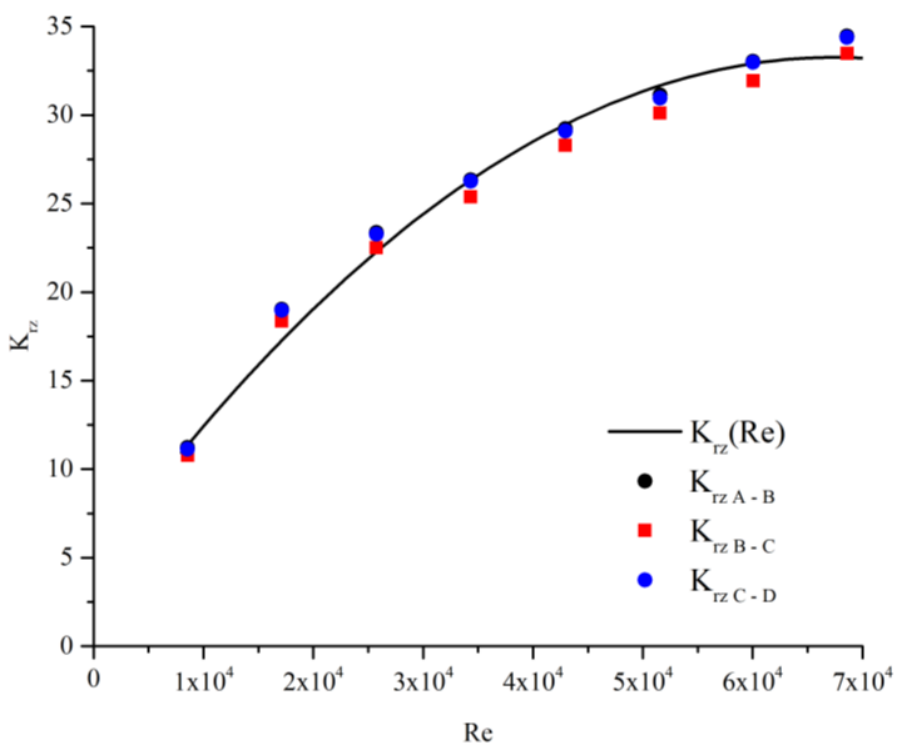

Figure 3. Friction anisotropy coefficient versus Re number.

\section{Conclusions}

The dependencies on the Reynolds number of the pressure drop and the coefficient of friction anisotropy in the transverse flow of a liquid in a rod bundle formed by hexagonal 
cylinders are obtained by using the CFD code CONV-3D based on the DNS approximation. This dependence can be recommended for use in thermal-hydraulic analysis codes that require simulation of the flow in similar structures, for example, in the inter-fuel-assembly space of the core of SFRs. It should be noted that further refinement of the obtained dependencies is possible on the basis of the stochastic relations presented in [7-9].

Author Contributions: Conceptualization, Y.E.S.; validation, M.V.B.; writing—original draft preparation, M.V.B. and R.P.D.; writing — review and editing, Y.E.S.; visualization, R.P.D.; project administration, Y.S.K. All authors have read and agreed to the published version of the manuscript.

Funding: This research received no external funding.

Institutional Review Board Statement: Not applicable.

Informed Consent Statement: Not applicable.

Acknowledgments: The authors are grateful to the staff of IBRAE: Vladimir V. Chudanov, Valery A. Pervichko, and Anna E. Aksenova for valuable advice on using the new generation CONV-3D code.

Conflicts of Interest: The authors declare no conflict of interest.

\section{References}

1. Evgenyevich, S.Y.; Alekseevich, K.I. Analytical studies on thermal-hydraulic parameters of fast reactor taking into account effect of inter-wrapper space. In Proceedings of the International Conference on Fast Reactors and Related Fuel Cycles: Safe Technologies and Sustainable Scenarios, FR-09, Kyoto, Japan, 7-11 December 2009.

2. Idelchik, I.E. Handbook of Hydraulic Resistance, 4th ed.; Research Institute for Gas Purification: Moscow, Russia, 2008; 861p.

3. Chudanov, V.V.; Aksenova, A.E.; Pervichko, V.A.; Makarevich, A.A. Supercomputer Mathematical Modeling of the Fluid Dynamics in Elements of Nuclear Power Facilities. At. Energy 2015, 117, 381-387. [CrossRef]

4. Chudanov, V.V.; Aksenova, A.E.; Makarevich, A.A.; Pervichko, V.A.; Romero-Reyes, I.V. Development of a Method of Direct Numerical Modeling of Turbulent Flows Using a Supercomputer. At. Energy 2015, 118, 247-253. [CrossRef]

5. Chudanov, V.V.; Aksenova, A.E.; Makarevich, A.A.; Pervichko, V.A.; Reyes, I.V.R. Use of the CFD-Code CONV-3D in Reactor Applications. At. Energy 2017, 121, 179-184. [CrossRef]

6. Chudanov, V.V.; Aksenova, A.E.; Pervichko, V.A. Modeling of Incompressible Fluid Flows in a Round Tube in Laminar, Transient, and Turbulent Regimes using the Conv-3d Code. At. Energy 2020, 127, 328-332. [CrossRef]

7. Dmitrenko, A.V. Determination of the Coefficients of Heat Transfer and Friction in Supercritical-Pressure Nuclear Reactors with Account of the Intensity and Scale of Flow Turbulence on the Basis of the Theory of Stochastic Equations and Equivalence of Measures. J. Eng. Phys. Thermophys. 2017, 90, 1288-1294. [CrossRef]

8. Dmitrenko, A.V. Results of investigations of non-isothermal turbulent flows based on stochastic equations of the continuum and equivalence of measures. J. Phys. Conf. Ser. 2018, 1009, 012017. [CrossRef]

9. Dmitrenko, A.V. Calculation of pressure pulsations for a turbulent heterogeneous medium. Dokl. Phys. 2007, 52, 384-387. [CrossRef] 